\title{
Diseño ergonómico de un dispositivo Braille para la lecto-escritura e impresión
}

\section{Design ergonomic of a device Braille for Reading-writing and printing}

\author{
CRUZ-SOLIS, Edgar Jesús†**, REYES-LEON, Iván, HERNÁNDEZ-LUNA, Aldo, y CRUZ-LUNA, \\ Manuel
}

Instituto Tecnológico Superior de Huauchinango

ID $1^{\text {er }}$ Autor: Edgar Jesús, Cruz-Solís / ORC ID: 0000-0003-4083-0888, Researcher ID Thomson: I-4334-2018, CVU CONACYT ID: 904718

ID $1^{\mathrm{er}}$ Coautor: Iván, Reyes-León / ORC ID: 0000-0003-1294-0772, Researcher ID Thomson: H-8715-2018, CVU: 903594

ID $2^{\text {do }}$ Coautor: Aldo, Hernández-Luna / ORC ID: 0000-0002-7717-5314, Researcher ID Thomson: I-1165-2018, CVU CONACYT ID: 441305

ID $3^{\text {er }}$ Coautor: Manuel, Cruz-Luna / ORC ID: 0000-0002-0640-8926, Researcher ID Thomson: H-8709-2018, CVU CONACYT ID: 368159

DOI: $10.35429 /$ JEA.2019.20.6.1.8

Recibido: 19 de Junio, 2019; Aceptado 30 de Septiembre, 2019

\section{Resumen}

En la humanidad existen diversas personas con discapacidad visual, denominando así ésta condición a la pérdida total o parcial de la vista. En la investigación realizada se presenta el diseño ergonómico de un dispositivo que se encuentra integrado por 4 sistemas escritura, lectura, tracción e impresión; el diseño del dispositivo se realizó mediante 2 fases, la primera corresponde al proceso de diseño de los sistemas mencionados anteriormente y la segunda al diseño ergonómico del dispositivo y control. Con la elaboración de éste diseño se pretende beneficiar a las personas que poseen discapacidad visual ayudándolos al proceso de su aprendizaje y así mismo desarrollar en estas personas diversas habilidades comunicativas, de igual forma se busca facilitar la lecto-escritura e impresión en sistema Braille con un diseño más ergonómico.

Ergonomía, Sistema Braille

\begin{abstract}
In humanity there are several people with visual impairment, thus calling this condition to the total or partial loss of sight. In the research carried out, the ergonomic design of a device that is integrated by 4 writing, reading, traction and printing systems is presented. The design of the device was made through 3 phases, the first corresponds to the design process of the systems mentioned above, the second to the ergonomic design of the device and control and the last to the integration process of all the systems described above. With the development of this design is intended to benefit people who have visual impairment by helping them to the process of their learning and also develop in these people various communication skills, with this device is intended to facilitate reading and writing with the Braille system for people with this disability and in the same way provide them with a more ergonomic and easy to use design.
\end{abstract}

Ergonomics, Braille system

Citación: CRUZ-SOLIS, Edgar Jesús, REYES-LEON, Iván, HERNÁNDEZ-LUNA, Aldo, y CRUZ-LUNA, Manuel. Diseño ergonómico de un dispositivo Braille para la lecto-escritura e impresión. Revista de Aplicaciones de la Ingeniería. 2019. 620: $1-8$

\footnotetext{
* Correspondencia del Autor (Correo electrónico: edgar.itsh@gmail.com)

$\dagger$ Investigador contribuyendo como primer autor.
} 


\section{Introducción}

La discapacidad visual es la condición que padecen algunas personas con una pérdida parcial de la vista, se les atañe este concepto debido a que quienes la padecen son capaces de distinguir ciertos objetos, a diferencia de las personas que padecen de ceguera, puesto que esta condición es caracterizada por la ausencia total de la capacidad visual, es decir, que éstas no son capaces de distinguir la luz de la oscuridad y por ende la adquisición de conocimiento por este sentido resulta imposible, es por ello que el hombre en respuesta a esta necesidad ha creado diversos mecanismos que ayudan a las personas que padecen de esta condición a poder comunicarse y así mismo a poder adquirir conocimiento.

Un sistema muy conocido para la comunicación es el sistema Braille que tuvo sus inicios en Francia a mediados del siglo XIX, cabe decir que sus fines inicialmente eran netamente militares, puesto que éstos encontraron un lenguaje para poder comunicarse en clave, algo que resulta muy importante mencionar es que la persona que diseño el primer prototipo fue un capitán llamado Charles Barbier y que tiempo después se dio cuenta que éste sistema podía ser utilizado por las personas con discapacidad visual o ciegas y fue así como llegó a manos de un alumno llamado Louis Braille, el cual a partir de distintas modificaciones creó un sistema universal.

El sistema Braille fue evolucionando con el pasar de los años, tanto así que ya para el año de 1878 se creó un decreto que establece al sistema Braille como universal y a raíz de ello se empezó a aplicar este sistema en otros países como Rusia, China, Japón y otros cuya descendencia era de lengua árabe. Un caso muy importante es el sucedido en Japón, el cual para la aplicación de este sistema se tuvo que crear un silabario al que llamaron Katakana y fue desarrollado por el profesor Kuraji Ishikawa con la colaboración de varios alumnos invidentes, con el tiempo este sistema se fue sofisticando aún más diseñando diferentes mecanismos que ayudan al entendimiento un poco más amplio sobre este.
Tanto así que ahora en la actualidad existen diversos dispositivos que les facilitan la vida a las personas que lo padecen y que para la creación de estos como es de esperarse varios investigadores realizaron estudios minuciosos sobre el comportamiento de las personas invidentes, que los llevaron a crear diferentes técnicas y procedimientos que hoy día se utilizan para ayudar a las personas con esta condición, al hecho mencionado anteriormente se le conoce hoy día como Tiflotecnología, que se apoya en la tiflomecánica, que se asocia más con los artefactos que se crean para ayudar a las personas ciegas o con discapacidad visual.

El primer dispositivo con este propósito fue la máquina de escribir Braille desarrollada a los principios del siglo XX y creada por Óscar Pich, quien tuvo la primera iniciativa de crear algún artefacto para las personas con la discapacidad visual; partiendo del modelo propuesto por Pich diversos autores se apoyaron en estos para crear nuevas y mejores máquinas del lenguaje Braille, tanto así que en la actualidad se han creado otros artefactos encaminados al mismo propósito como lo es la impresora Braille.

Aunque existen diversos dispositivos que ya resuelven o suplen las necesidades de la población invidente es claro que la preocupación ahora va un poco más allá, orientándose un poco más a la comodidad y a la ergonomía de los diseños, pensando principalmente en lo dicho es que en ésta investigación se propone un nuevo diseño con características más particulares, en específico un diseño ergonómico y la integración de 4 sistemas generales de funcionamiento, uno de escritura mediante el uso de un control, otro de lectura en el que se muestra la letra, el número o el carácter que es escrito por medio del control, éste sistema le proporciona al usuario la oportunidad de verificar si efectivamente lo escrito es correcto; un sistema de tracción encargado de proporcionarle el movimiento de la hoja para el ingreso del dispositivo y por último el de impresión en el que se accionará una vez que el usuario haya verificado lo escrito, se le da la opción de imprimir el texto escrito.

Teniendo en cuenta lo dicho y bajo la misma línea, es preciso afirmar que ésta investigación puede provocar un gran impacto en toda la comunidad invidente. 
Puesto que con éste nuevo diseño además de proporcionarles acceso a las distintas culturas también representa una forma más fácil de poder penetrar al mundo del conocimiento y hacerlo en un menor tiempo posible, apuntándole a la reducción del tiempo empleado para aprender a usar éste sistema, además de lo dicho éste diseño presenta una estructura más ergonómico pensando en la comodidad de los usuarios y por tanto en su salud.

Es bien sabido que lo largo de la historia han existido como ya se mencionó diversos mecanismos que han tratado de dar respuesta a las necesidades de las personas invidentes, pero que se han centrado básicamente en esta prioridad y no mucho en la ergonomía, lo cual resulta un factor muy importante a tener en cuenta para la fabricación de los dispositivos, ya que un diseño en el que no se tiene en cuenta la ergonomía, es un diseño que puede ocasionar efectos negativos a las personas que los usan, en los distintos dispositivos que existen para la escritura del lenguaje Braille son como teclados parecidos a los de las computadoras, por ende se podría decir que este diseño si se usa de forma muy periódica puede desarrollar distintas enfermedades como el síndrome del túnel carpiano, la artritis, la Teno sinovitis o lesiones en la mano y muñeca como el del dedo engatillado, dichos efectos se pueden reducir diseñando un mejor mecanismo que sea más adaptable a la estructura de las manos de las personas invidentes.

A lo largo del presente escrito se detallará cada uno de los sistemas que conforman el diseño ergonómico del dispositivo tiflotécnico de lecto-escritura con impresión Braille.

\section{Descripción metodológica}

El diseño general como se mencionó anteriormente consta de 4 sistemas generales de funcionamiento, los cuales son escritura mediante el uso de un control, lectura, impresión y por último de tracción de hoja y para el respectivo diseño de estos sistemas el proceso se dividió en 2 fases.
La primera de éstas está dedicada a el proceso de diseño de todos los sistemas que conforman el diseño general los cuales son el de escritura, lectura, tracción e impresión y en la segunda como parte de los resultados se describe el proceso del diseño ergonómico tanto del dispositivo como el de control, las fases mencionadas anteriormente se pueden sintetizar tal como se puede observar en la figura 1 .

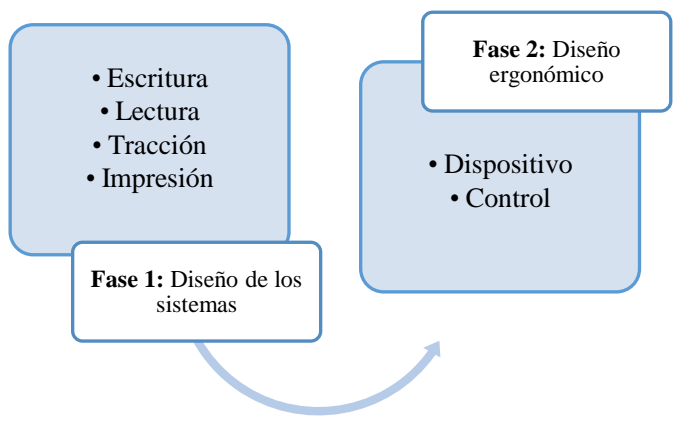

Figura 1 Fases de la metodología

Fuente: María Luisa Castro Sánchez

Partiendo de la descripción de cada una de las fases descritas anteriormente a continuación, se procede al desarrollo de cada uno de las fases.

\section{Resultados}

\section{Aplicación de la fase 1: Desarrollo de todos los sistemas}

En esta fase se describe de forma detallada cada uno de los desarrollos de los distintos sistemas que conforman al dispositivo los cuales son el de escritura, lectura, tracción e impresión

\section{Sistema de escritura}

El mecanismo de funcionamiento de éste sistema se centra principalmente en la estructura del control de escritura, el cual está conectado por medio de un cable al dispositivo general, este artefacto consta de 8 botones distribuidos a lo largo de todo el control, 6 de ellos harán el papel de los cuadratines del sistema Braille y la distribución de éstos mismos consta de tres botones en la parte derecha trasera del control y los restantes en la izquierda trasera del mismo, cada botón representa la ubicación de los dedos tanto de la mano derecha como de la mano izquierda, es decir, que los primeros botones de arriba hacia abajo corresponderán a los dedos índices, el segundo a los dedos corazón y el tercero a los dedos anular. 
Por último están los dos botones restantes que serán usados por los dedos pulgares, el botón con el que será presionado por el pulgar derecho tendrá la función de borrar la última letra y el que será presionado por el pulgar izquierdo tendrá la función de la barra espaciadora.

\section{Sistema de lectura Braille}

El funcionamiento del sistema de lectura Braille se basa en un mecanismo de forma lineal horizontal que se compone de dos engranes, uno en el que se encuentra conectado a un servomotor que le proporciona el movimiento, éste artefacto tiene una rotación gradual de $360^{\circ}$, es importante mencionar que su estructura interna la conforma una parte electrónica (Controlador), una eléctrica (Motor) y una parte mecánica que la compone una caja de engranes, este aparato está conectado a una tarjeta arduino por medio de 3 cables, uno positivo otro negativo y uno de señal, este último es el responsable de proporcionarle el ángulo de inclinación que tendrá el engrane que se traduce en un movimiento rotatorio, cabe recalcar que dependiendo la modulación de ancho de pulso que se le suministre al cable éste reflejará un ángulo que a su vez se traduciría en un movimiento con un sentido ya sea horario o anti horario, dicho movimiento también dependerá del ángulo estándar del sistema, cabe decir que el movimiento rotatorio que tiene el engrane impulsado por el servomotor se traduce en una respuesta netamente lineal, puesto que se encuentra en sincronía con una placa recta con pequeños abultamientos los cuales le proporcionan a unas barras pequeñas, el movimiento de forma vertical trabajando este movimiento en el eje positivo de las z los cuales estarán de forma perpendicular a la superficie de la placa, provocando así la manifestación en el cuadratín de la letra, el número o el carácter que la persona haya digitado. La estructura del sistema que se describió anteriormente se muestra en la figura 2

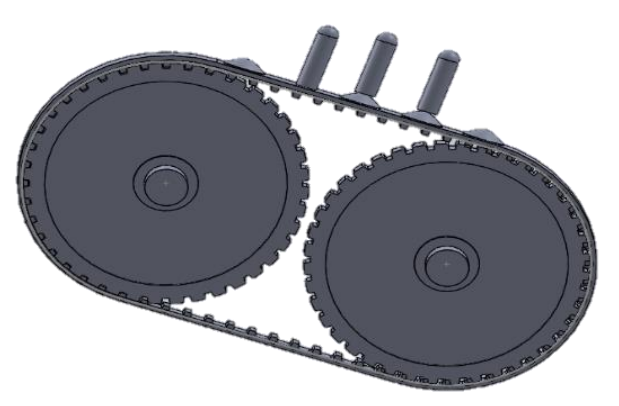

Figura 2 Diseño del sistema de lectura

Fuente: Ulises Barzalobre Cruz

\section{Sistema de tracción de hoja}

El funcionamiento del sistema de tracción de la impresora Braille se basa en un mecanismo en el que se apoya de dos pares de rodillos que se encuentran unidos por un eje, los cuales son los responsables del movimiento de la hoja, el primer par de rodillos entra en contacto con la hoja y la sujeta en conjunto con la base para generar la tracción, seguidamente la hoja pasa por la base principal de escritura y justamente en esta área se encuentran 4 micro rodillos que se encargan del soporte de la hoja, para impedir que ésta se mueva, luego de éste proceso la hoja finalmente llega a el segundo par de rodillos para proporcionarle la salida, es importante decir que tanto el primer como el segundo par de rodillos se encuentran sincronizados, puesto que éstos se encuentran conectados por una banda de hule que es accionada por un motor para generar el movimiento.

Bajo el mecanismo de funcionamiento de este sistema se procedió al diseño general teniendo de criterios muy particulares como el tamaño de la hoja sobre el que normalmente las personas con discapacidad visual trabajan, la cantidad de componentes del sistema, su configuración, la función general del sistema mecánico y las condiciones estructurales de la sujeción de los componentes que integran el sistema de tracción de la hoja.

En las siguientes figuras se describirán a detalle cada uno de los componentes que integran el sistema de tracción. En la figura 3 se muestra la estructura principal del sistema mencionado anteriormente, el cual lo componen el par de rodillos unido por los ejes y la base del chasis.

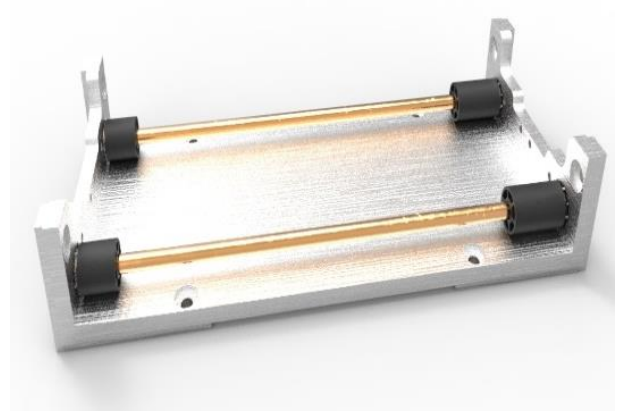

Figura 3 Diseño del sistema de tracción de la hoja Fuente: Víctor Manuel Ventura Ahumada 
En la figura 4 se puede observar con más detalle la estructura de los pares de rodillos que se encuentra en el sistema de tracción, es importante mencionar que en la figura se ilustra solo el par de rodillos principales, que en forma y estructura resulta igual que el de los secundarios.

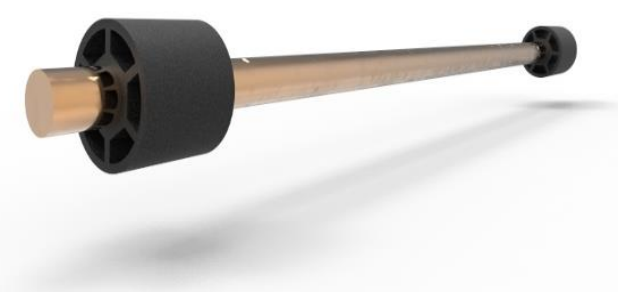

Figura 4 Par de rodillos principales conectados por el eje Fuente: Víctor Manuel Ventura Ahumada

En la figura 5 se muestra las partes del micro rodillo que se encuentran en los costados del área de escritura con vista isométrica. La parte que se encuentra de color negro representa el micro rodillo y la de color azul representa la estructura principal de soporte para los rodillos con empotre al chasis.

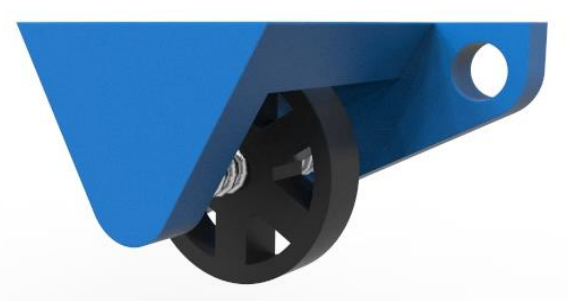

Figura 5 Parte de un micro rodillo desde una vista isométrica

Fuente: Víctor Manuel Ventura Ahumada

En la figura 6 se muestra la misma estructura, pero vista de forma frontal en la cual se puede ver que el resorte hace la función de eje del micro rodillo, representado por el color plateado, este posee una forma helicoidal

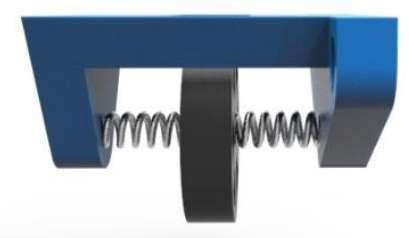

Figura 6. Parte de un micro rodillo visto de forma frontal Fuente: Víctor Manuel Ventura Ahumada

En la figura 7 que se encuentra a continuación se muestra la descripción particular de la rampa del sistema de tracción, esta parte consta de dos bases laterales y una parte curva de acrílico.

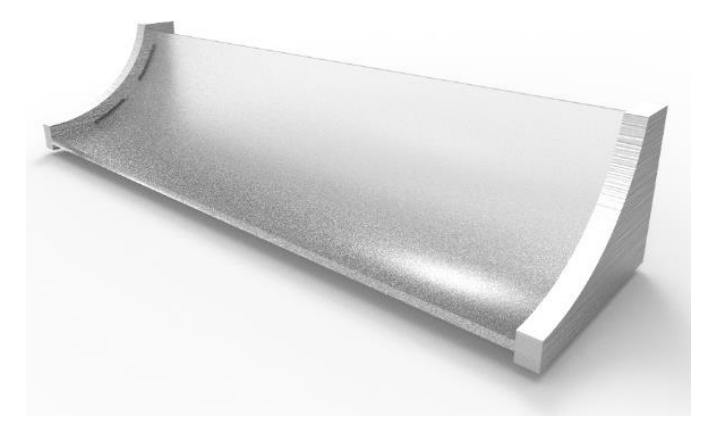

Figura 7 Estructura de la rampa Fuente: Víctor Manuel Ventura Ahumada

Todos los esquemas que se presentan en las figuras anteriores se unieron para formar el sistema de tracción el cual se encuentra ilustrado en la figura 8 , es importante mencionar que los colores que se da a conocer en la imagen son caracteristicas especiales del material del cual es la pieza. Las piezas azules que son la de soporte para los micro rodillos son de PLA que es un ácido poli-láctico, material polímero biodegradable derivado del ácido láctico, del material mencionado anteriormente también se encuentra hecho los Rodillos y los micro rodillos, la única diferencia es el color del PLA, pero el material es igual, el eje sobre el que están los pares de rodillos es de acero inoxidable al bajo carbono y el resto de las partes del sistema es de aluminio pulido.

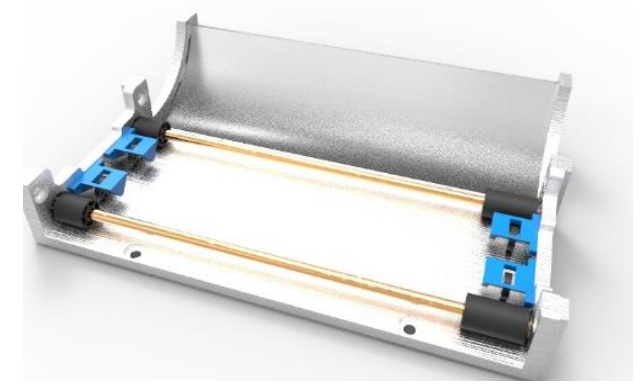

Figura 8 Estructura general del sistema de tracción de hoja hecho en el software Keyshot

Fuente: Víctor Manuel Ventura Ahumada

En la siguiente imagen se representa el mismo sistema renderizado solo que desde una vista frontal tal como se representa en la figura 9

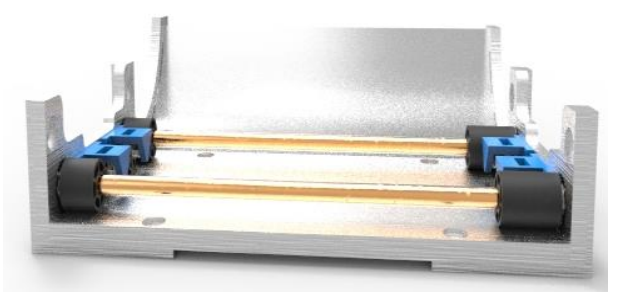

Figura 9 Estructura general del sistema de tracción de hoja hecho en vista frontal

Fuente: Víctor Manuel Ventura Ahumada 


\section{Sistema de impresión de la hoja}

El mecanismo de funcionamiento de éste sistema se encuentra conformado por tres partes que son: el recorrido de la banda de hule, el punzón y la estructura de soporte del punzón, los tres componentes mencionados se colocan en funcionamiento por el eje de un motor, ya que es éste el que le va proporcionar el movimiento primeramente a la banda y que a su vez accionará la estructura de soporte y de la misma manera provocará el movimiento al solenoide que es dónde se encuentra el punzón, accionado este ya se encuentra listo para empezar a escribir, es importante recalcar que éste último solo se moverá en el eje de las $x$.

En esta etapa se muestra el prototipo final en el cual se integran todos los sistemas que lo integran ya mencionados anteriormente, obteniendo el dispositivo y control de manera integral, en la fase 2 se muestra a detalle.

\section{Aplicación de la fase 2: Diseño ergonómico}

Teniendo en cuenta los sistemas descritos anteriormente y partiendo de la ubicación espacial de cada sistema se diseña la estructura ergonómica tanto del dispositivo como del control de escritura

\section{Dispositivo}

Para el diseño de la estructura que cubrirá el dispositivo particular se tomaron criterios muy puntuales sobre el espacio que ocupan cada uno de los diversos sistemas específicamente el de lectura, tracción e impresión de hoja. Este diseño lo conforma la parte de salida de las barras las cuales formaran la letra escrita con el control, ésta zona se encuentra en la parte superior de la carcasa de tal manera que se ajusta perfectamente al brazo de la persona invidente para que ésta pueda leer, además de lo dicho éste dispositivo consta de dos compuertas, una por donde el cual ingresan las hojas y la otra para la salida de la hoja, siguiendo la misma línea éste diseño cuenta con un puerto USB y una pantalla para que las personas que acompañan a las personas invidentes puedan observar lo escrito en castellano o inglés según el idioma que manejen la persona.

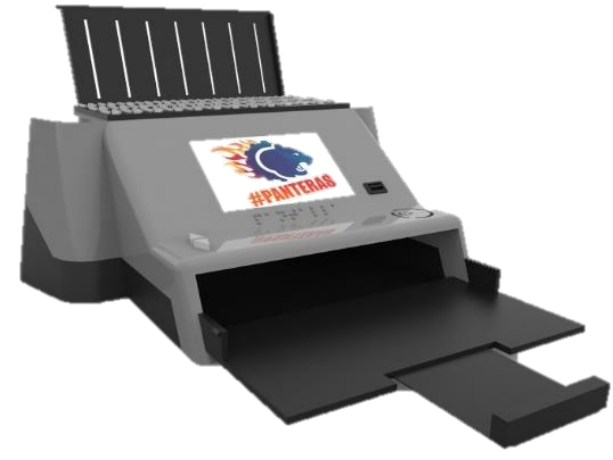

Figura 10 Estructura del diseño ergonómico del dispositivo en particular

Fuente: Víctor Manuel Ventura Ahumada

\section{Control}

Para la debida estructura y diseño del control se realizó un estudio tomando una muestra al azar de 15 mujeres y 15 hombres con el objeto de tomarle las mediciones de acuerdo a las medidas antropométricas y también a los criterios propios de diseño. Para estas medidas se establecieron percentiles muy puntuales, para la largura de la mano cuya medida va desde el final de la palma hasta el comienzo del dedo medio, la largura de la palma y la altura de ésta misma, se establecieron unos percentiles de 90, seguidamente para el diámetro de agarre y la distancia del final del pulgar hasta el final del dedo índice su percentil fue de 20, de ésta misma forma la largura del dedo índice, medio, anular y pulgar tuvieron un percentil de 30, es importante mencionar que estas mediciones se hicieron tanto para la mano derecha como para la izquierda y con base a ella se diseñó el diseño ergonómico que se puede ver en la figura 10.

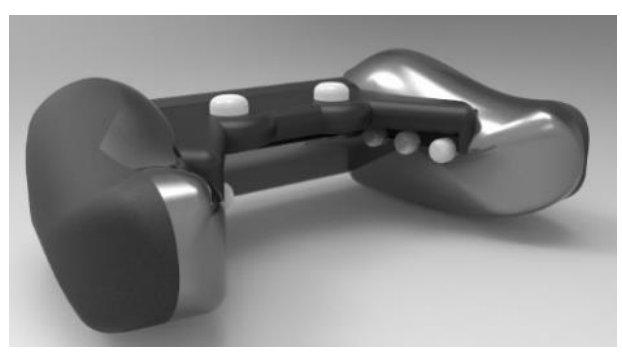

Figura 10 Diseño ergonómico del control Fuente: Víctor Manuel Ventura Ahumada 


\section{Anexos}

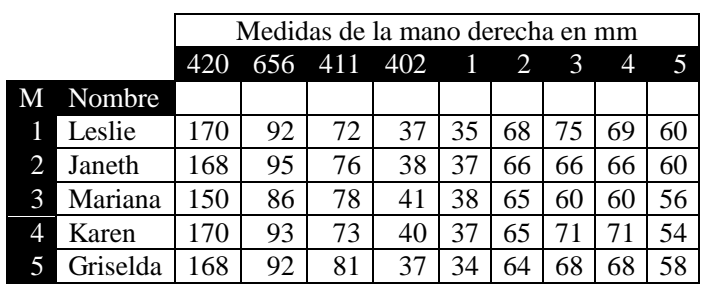

Tabla 1 Toma de muestras de mano derecha mujeres Fuente: María Luisa Castro Sánchez

\section{Agradecimiento}

A la carrera de Ingeniería Industrial del Instituto Tecnológico Superior de Huauchinango por el apoyo y las facilidades en el desarrollo del proyecto, así mismo este artículo fue realizado como producto de la colaboración interinstitucional entre los cuerpos académicos de Tecnología Aplicada del ITSH y Automatización y Control de Procesos del ITSH, así como con la carrera de Ingeniería Eléctrica del ITSH.

De igual manera un agradecimiento al programa Delfín y por supuesto a los alumnos que fueron participes en el desarrollo de este proyecto: Oscar Antonio Aguayo Tut de la Universidad de Quintana Roo, Víctor Manuel Ventura Ahumada de la Facultad de Arquitectura y Diseño, Universidad de Colima, María Luisa Castro Sánchez de Corporación Universitaria del Caribe CECAR, Colombia y Ulises Barzalobre Cruz del Instituto Tecnológico Superior de Huauchinango.

\section{Conclusiones}

Después del desarrollo de la investigación realizada, se puede concluir que para realizar un diseño ergonómico de cualquier mecanismo se debe tener en cuenta el objetivo de su funcionamiento y la comodidad que debe proporcionar, tal como se pudo evidenciar al momento de diseñar el control y la carcasa del dispositivo, puesto que se tuvo en cuenta su utilidad y ciertos parámetros que se apoyaron de la antropometría.

\section{Comentarios finales}

El trabajo desarrollado aporta un avance científico en el área debido a que los resultados obtenidos dan a conocer un diseño ergonómico innovador, el cual integra los sistemas de lectura, escritura, impresión y tracción, integración que en la actualidad no existe, es importante mencionar que los resultados obtenidos se relacionan directamente con lo expuesto en el objetivo general, el cual es diseñar de forma ergonómica el control y el dispositivo tiflotécnico de lecto-escritura con impresión Braille.

Para el debido cumplimiento de éste objetivo se aplicó una metodología que consta de dos fases, en la primera se desarrolló el diseño general de los 4 sistemas de funcionamiento escritura, lectura, impresión y tracción y en la segunda el diseño ergonómico del control y la cubierta del dispositivo, cabe decir que en ésta última fase, para el diseño del control se realizó una toma de datos a una muestra conformada por 15 mujeres y 15 hombres, de los cuales se tomaron medidas muy puntuales como lo fue el diámetro de agarre, la anchura y largura de la mano y la longitud de los dedos, tanto de la mano derecha, como de la izquierda, es importante mencionar que el instrumento utilizado para las mediciones descritas anteriormente fueron hechas con un calibrador Vernier y las medidas fueron registradas en una tabla, la cual se encontraba constituida por 11 columnas en las que se encontraban descritas los nombres de las personas, y las características de la medición.

\section{Referencias}

Análisis Multivariante de los Aspectos Emocionales y las Inteligencias Múltiples en la Era Digital. Edição/Edition, 234.

Cisneros, J. C., Chimbo, K. M. O., Trejo, C. A., Valdez, K. G. E., \& Villardón, J. L. V. (2019). García Hernández, M. N., Palacios, J., Isaías, A., \& Cerezo Alvarez, E. (2019). Diseño e implementación de un sistema detector ultrasónico de obstáculos con aviso de tonos para personas con baja visión.

González, R. M. La enseñanza de los instrumentos de cuerda frotada en la práctica de aula: 15 años de tesis doctorales españolas. Revista Electrónica de LEEME, 1(43). 
Pérez Sánchez, A. M. (2019). Dificultades de Aprendizaje y Trastornos del Desarrollo. Educación Primaria. Apuntes (2019). Dificultades de Aprendizaje y Trastornos del Desarrollo.

Riera, X. (2019). Historia de la Tiflotecnología en España. [online] Nosolousabilidad.com. Available at: http://www.nosolousabilidad.com/articulos/tiflo tecnologia.htm [Accessed 27 Jun. 2019].

Sastre Caicedo, D. L. Plan de mejoramiento de los servicios que presta la biblioteca pública municipal Joaquín Piñeros Corpas, para personas con discapacidad visual.

Torres, A. (2019). La historia del Braille, un alfabeto accesible. [Online] Periodico.laciudadaccesible.com. Available at: http://periodico.laciudadaccesible.com/portada/ opinion-la-ciudad-accesible/item/4605-lahistoria-del-braille-un-alfabeto-accesible [Accessed 27 Jun. 2019].

Villalobos, F., Esparza, M. and Muñoz, J. (2019). Diseño de Impresora Braille a Partir de Impresora de Matriz de Puntos. [online] Redalyc.org. Available at: http://www.redalyc.org/jatsRepo/944/94457671 008/html/index.html [Accessed 27 Jun. 2019]. 